Neurosurg Focus 18 (6b):E4, 2005

\title{
Instructional video
}

\author{
Dissection of the human brain
}

\author{
LenNart Heimer, M.D. \\ Department of Neurological Surgery, University of Virginia, Charlottesville, Virginia
}

KEY WORDS • dissection - brain anatomy - instructional video

No textbook or model can replace dissection of the human brain as the ultimate educational experience. To take full advantage of the dissection, however, it must be carefully planned. The purpose of this DVD is to serve as a practical guide for the systematic dissection of the human brain. It also emphasizes the technique of blunt dissection for the study of major fiber bundles.

To encourage a more general use of brain dissection as an educational device, it should be mentioned that no elaborate pretreatment of the organ is needed. The brains used for this DVD were removed from the skull 12 to 18 hours after death and fixed for 2 weeks in $20 \%$ buffered formalin and then in $10 \%$ formalin for several weeks to more than 1 year. To prevent distortion of the brains, they were suspended by a string fastened around the basilar artery or wrapped in gauze. They were washed overnight in running water before being dissected. None of the brains exhibited in this DVD was subjected to the Klingler ${ }^{2}$ freeze-thaw method following formalin fixation, which has been popularized as the pretreatment of choice for dissection of fiber tracts. ${ }^{3}$ There are a couple of reasons for not using the Klingler method as a routine procedure, unless the purpose of the dissection is focused entirely on white matter dissection.

The freeze-thaw technique developed by Klingler is based on the formation of ice crystals, which facilitate the separation of fiber bundles in the myelinated fiber tracts, which in turn makes it easier to demonstrate regions of cleavage within these tracts. The freeze-thaw technique, however, is not without its problems. The freezing of the tissue tends to affect the overall condition of the specimen. Deterioration of the distinction between gray and white matter or the occurrence of tissue fissures ${ }^{1}$ cannot always be avoided, which tends to increase the number of brains needed for dissection. This is an important consideration because an oversupply of brains for dissections is seldom available.

An additional reason for not using the Klingler method as a routine procedure is provided by numerous examples in the literature and by the pictures in this DVD, which indicate that the major fiber bundles can be clearly demonstrated in formalin-fixed brains without freeze-thaw pretreatment. In other words, unless the purpose of the dissection is the preparation of exhibition or museum specimens demonstrating major fiber bundles, there is no need for freeze-thaw pretreatment.

Whatever angle one may have for studying the structure of the human brain, its complicated anatomy often seems to be an obstacle. This may be the case especially in regard to brain dissections, which cannot be properly performed in an organized fashion without careful planning. In fact, it appears that disappointments with the results of these dissections are more often related to the lack of thoughtful planning and a cautious approach than to the specific pretreatment of the specimen. It is in this spirit that we have chosen to promote the general approach and technique of brain dissection described in a stepwise fashion in this DVD.

To illustrate the general technique presented in the DVD, I have chosen Section 3, in which the dissection of the temporal lobe is described.

\section{References}

1. Kier EL, Staib LH, Davis LM, et al: Anatomic dissection tractography: a new method for precise MR localization of white matter tracts. AJNR 25:670-676, 2004

2. Klingler J: Erleichterung der makroskopischen Präparation des Gehirns durch den Gefrierprozess. Schweiz Arch Neurol Psychiat 36:247-256, 1935

3. Yaşargil MG, Türe U, Yaşargil DC: Impact of temporal lobe surgery. J Neurosurg 101:725-738, 2004

Manuscript received May 4, 2005.

Accepted in final form May 30, 2005.

Address reprint requests to: Lennart Heimer, M.D., Department of Neurological Surgery, P.O. Box 800212, University of Virginia Health System, Charlottesville, Virginia 22908. e-mail: 1h2c@ virginia.edu. 
Dissection of the Human Brain on DVD (total playing time: 1 hour, 16 minutes)

Section 1. Dissection of the Cerebral Hemisphere From the Medial Side (27 mins)

Section 2. Dissection of the Cerebral Hemisphere From the Lateral Side (14 mins)

Section 3. Dissection of the Temporal Lobe (18 mins)

Section 4. Dissection of the Cerebellum and Brainstem (17 mins)

Section 1. Dissection of the Cerebral Hemisphere From the Medial Side.

Technique of Blunt Dissection

Overview of Structures to be Exposed
Cingulum and Related Structures

Lateral Ventricle and Related Structures

Internal Capsule

Superior Occipitofrontal Fasciculus: A Case of Mistaken Identity?

Section 2. Dissection of the Cerebral Hemisphere From the Lateral Side

Overview of Structures to be Exposed Insula

Superior Longitudinal Fasciculus

Extreme Capsule, Claustrum, and External Capsule

Section 3. Dissection of the Temporal Lobe

Occipitofrontal, Uncinate, and Inferior Longitudinal Fasciculi Optic Radiation and Meyer's Loop

Anterior Commissure, Meyer's Loop, and Internal Capsule

Lateral Ventricle, Hippocampus, and Amygdala

Section 4. Dissection of the Cerebellum and Brainstem

Cerebellum and Brainstem: Orientation

Cerebellar Peduncles

Flocculonodular Lobe and Rhomboid Fossa

Cerebellar Nuclei 